\title{
MORITA EQUIVALENCE AND DUALITY
}

\author{
Albert Schwarz
}

\begin{abstract}
It was shown by Connes, Douglas, Schwarz [1] that one can compactify $\mathrm{M}$ (atrix) theory on noncommutative torus $T_{\theta}$. We prove that compactifications on Morita equivalent tori are in some sense physically equivalent. This statement can be considered as a generalization of nonclassical $S L(2, \mathbb{Z})_{N}$-duality conjectured in [1] for compactifications on twodimensional noncommutative tori.
\end{abstract}

\section{Introduction.}

Methods of noncommutative geometry have important applications to M(atrix) theory. First application of this kind was given in [1], where these methods were used to describe a new type of toroidal compactifications of $\mathrm{M}$ (atrix) theory. Later such compactifications (compactifications on noncommutative tori) were studied in numerous papers (see for example [2]).

In present paper we apply to physics the notion of Morita equivalence of algebras that plays very significant role in noncommutative geometry. Namely, we prove that the consideration of Morita equivalent noncommutative tori leads to a kind of duality of compactifications of $\mathrm{M}$ (atrix) theory that generalizes nonclassical $S L(2, \mathbb{Z})_{N}$-duality conjectured in [1] for two-dimensional case. Our analysis of this duality is based on mathematical results about Morita equivalence of multidimensional noncommutative tori obtained in the paper [3] and in this paper.

We work in the framework of IKKT M(atrix) theory [9]; however by means of Wick rotation one can obtain corresponding results in BFSS M(atrix) model [8].

Our main results are as follows. We show that compactifications on $n$ dimensional noncommutative tori $T_{\theta}$ and $T_{\widehat{\theta}}$ are physically equivalent if antisymmetric matrices $\widehat{\theta}$ and $\theta$ are related by the formula $\widehat{\theta}=g \theta=(A \theta+B)(C \theta+D)^{-1}$ where the block matrix $g$ consisting of $n \times n$ matrices $A, B, C, D$ belongs to the group $S O(n, n \mid \mathbb{Z})$. "Vector bundles" (projective modules) over noncommutative tori (as well as vector bundles over commutative tori) can be described by means of integer valued antisymmetric tensors of even rank, i.e. by even integer elements of fermionic Fock space. Physically equivalent "vector bundles" over $T_{\theta}$ and $T_{g \theta}$ are related by linear canonical transformation in fermionic Fock space; this transformation corresponds to $g \in S O(n, n \mid \mathbb{Z})$. We can consider the 
space of "vector bundles" over all noncommutative tori; this is a total space of a fibration with a discrete fibre and a base consisting of all antisymmetric $n \times n$ matrices $\theta$. It follows from our results that monodromy in this fibration generates physical equivalence of "vector bundles".

In [4] we constructed noncommutative instantons in $\mathbb{R}^{4}$ and formulated a generalization of Nahm duality for instantons on four-dimensional noncommutative torus (see [5] for details). It is mentioned in[4] that Nahm duality is related to Morita equivalence; we are planning to return to this relation later.

The results of noncommutative geometry that we use are explained in the paper. The reader willing to learn more about these results can find necessary information in Connes' book [6] and in papers [7].

Appendix contains some information about $K$-theory and remarks about relation of $K$-theory to matrix models. It is well known that in $M$-theory one should consider not only vector bundles, but also more general objects; it seems that our remarks give an explanation of this fact.

\section{Basic notions.}

Let us consider a pre- $C^{*}$-algebra $A$ (i.e. an associative algebra over complex numbers with a norm \|\| and antilinear involution $*$ obeying the condition $(x y)^{*}$ $\left.=y^{*} x^{*},\left\|x x^{*}\right\|=\|x\|^{2}\right)$.

Let $E$ be a right $A$-module equipped with $A$-valued inner product $\langle\xi, \eta\rangle$ that obeys $<\xi, \eta a>=<\xi, \eta>a,<\xi, \eta>^{*}=<\eta, \xi>,<\xi, \xi>$ is a positive element of $A$. (Here $\xi, \eta \in E, a \in A$.) Such a module is called a pre- $C^{*}$ module. $C^{*}$-algebras and $C^{*}$-modules are defined as complete pre- $C^{*}$-algebras and pre- $C^{*}$-modules. We will work with these objects taking completions of pre- $C^{*}$-algebras and modules if necessary.

It follows from the definition of inner product that the expression $f(\eta)=<$ $\xi, \eta>$ is an $A$-linear map from $E$ into $A$. We will assume that every $A$-linear map from $E$ into $A$ can be represented in this form. An endomorphism of a $C^{*}$-module $E$ is by definition an $A$-linear map $T: E \rightarrow E$ having an adjoint $\operatorname{map} T^{*}$ (i.e. $T(\xi a)=T(\xi) \cdot a,<\xi, T \eta>=<T^{*} \xi, \eta>$, where $\left.\xi, \eta \in E, a \in A\right)$. One can check that the algebra $\operatorname{End}_{A} E$ of endomorphisms of $E$ is a $C^{*}$-algebra with respect to the operator norm and involution $T \rightarrow T^{*}$.

If $E$ is a finitely generated free module $A^{n}$ (direct sum of $n$ copies of $A$ ), then endomorphisms can be identified with $n \times n$ matrices with entries from $A$.

In what follows $A$ always stands for a $C^{*}$-algebra having a unit. We will consider only finitely generated projective $C^{*}$ - modules (i.e. A-modules that can be represented as direct summands in free modules $\left.A^{n}\right)$. Then an endomorphism of an $A$-module $E$ can be defined as any map $T: E \rightarrow E$ obeying $T(\xi a)=T(\xi) a$; the condition that $T^{*}$ exists is satisfied automatically.

By definition a $C^{*}$-algebra $B$ is (strongly) Morita equivalent to the $C^{*}$ algebra $A$ if it is isomorphic to the algebra $\operatorname{End}_{A} E$ for some full module $E$. (One says that $E$ is full if the linear span of the range of inner product $\langle\xi, \eta\rangle$ 
is dense in $A$. Then one can prove that this linear span coincides with $E$. For important case when $A$ is a noncommutative torus all modules are full.)

There exists another definition of Morita equivalence that is less constructive, but much more useful in theoretic considerations. Let us consider two $C^{*}$ algebras $A, \hat{A}$ and an $(A, \hat{A})$-bimodule $P$. In other words we assume that the elements of $P$ can be multiplied by elements of $A$ from the left and by elements of $\hat{A}$ from the right, that $(a \xi) \hat{a}=a(\xi \hat{a})$ for $a \in A, \hat{a} \in \hat{A}, \xi \in P$ and that $P$ with these operations can be considered as a left $A$-module and right $\hat{A}$-module. As an $A$-module $P$ can be equipped with $A$-valued inner priduct $<,>_{A}$; as an $\hat{A}$-module it can be equipped by $\hat{A}$-valued inner product $<,>_{\hat{A}}$; we require that

$$
<\xi, \eta>_{A} \zeta=\xi<\eta, \zeta>_{\hat{A}} .
$$

We say that $P$ is an $(A, \hat{A})$-equivalence bimodule if the linear span of the range of $A$-valued inner product $<,>_{A}$ is dense in $A$ and the linear span of the range of $\hat{A}$-valued inner product $<,>_{\hat{A}}$ is dense in $\hat{A}$.

One says that $A$ and $\hat{A}$ are Morita equivalent if there exists an $(A, \hat{A})$ equivalence bimodule $P$. To relate this definition to the definition above we notice that the algebra $\operatorname{End}_{\hat{A}} P$ of endomorphisms of $P$ considered as $\hat{A}$-module is isomorphic to $A$. Conversely, every full right $A$-module $E$ can be considered as $\left(\right.$ End $\left._{A} E, A\right)$ - equivalence bimodule.

If $E$ is a right $A$-module and $P$ is an $(A, \hat{A})$ equivalence bimodule we define a right $\hat{A}$-module $\hat{E}$ by the formula

$$
\hat{E}=E \otimes_{A} P .
$$

Here the symbol $\otimes_{A}$ denotes the tensor product over $A$. (One can obtain $E \otimes_{A} P$ from the standard tensor product over $\mathbb{C}$ by means of identification $(\xi a) \otimes \eta \sim \xi \otimes(a \eta)$. Multiplication by $\hat{a} \in \hat{A}$ in $\hat{E}$ is defined by the formula $(\xi \otimes \eta) \hat{a}=\xi \otimes(\eta \hat{a})$; this definition is compatible with the above identification.)

If $P$ is an $(A, \hat{A})$-bimodule then complex conjugate linear space $\bar{P}$ can be considered as $(\hat{A}, A)$-bimodule. (We use formulas $\hat{a} \bar{x}=\overline{x \hat{a}^{*}}$ and $\bar{x} a=\overline{a^{*} x}$ to define the multiplication on $\hat{a} \in \hat{A}$ and $a \in A$ in $P$.) In the case when $P$ is an $(A, \hat{A})$ equivalence bimodule, one can prove that $\bar{P}$ is an $(\hat{A}, A)$ equivalence bimodule. Using $\bar{P}$ we can assign $A$-module to every $\hat{A}$-module; it is easy to prove that this operation is inverse to the correspondence $E \rightarrow \hat{E}$ and therefore the correspondence $E \rightarrow \hat{E}$ can be used to identify equivalence classes of $A$-modules and equivalence classes of $\hat{A}$-modules. The proof is based on the relation $P \otimes_{\hat{A}} \bar{P}=A$ where $A$ is considered as an $(A, A)$-bimodule.

It follows immediately from the definition that to every endomorphism $\alpha \in \operatorname{End}_{A} E$ one can assign an endomorphism $\widehat{\alpha} \in \operatorname{End}_{\hat{A}} \hat{E}$ as a map $\widehat{\alpha}: \hat{E} \rightarrow \hat{E}$ induced by a map $\alpha \otimes 1: E \otimes_{\mathbb{C}} P \rightarrow E \otimes_{\mathbb{C}} P$. (Moreover, one can say that the correspondence $E \rightarrow \hat{E}$ can be considered as equivalence of the category $A$-modules and the category $\hat{A}$-modules.) 
If an algebra $A$ is equipped with a trace $\operatorname{Tr}_{A}$ then using inner products in $(A, \hat{A})$-equivalent bimodule $P$ we can introduce a trace in $\hat{A}$ by the formula

$$
\operatorname{Tr}_{\hat{A}}<\xi, \eta>_{\hat{A}}=\operatorname{Tr} \quad{ }_{A}<\eta, \xi>_{A}
$$

where $\xi, \eta \in P$. (Recall that linear span of inner products $\langle\xi, \eta\rangle$ is dense in $\hat{A}$ by assumption and, moreover, one can prove, that it coincides with $\hat{A}$.) Similarly one can define a trace of endomorphism $\alpha \in \operatorname{End}_{A} E$; one can prove that

$$
\operatorname{Tr} \widehat{\alpha}=\operatorname{Tr} \alpha .
$$

Notice that in the case when the trace on $A$ is normalized (i.e. $\operatorname{Tr} 1=1$ ) the corresponding trace on $\operatorname{End}_{A} E$ is not necessarilly normalized; its value on unit element is the dimension of $A$-module $E$. (One can consider this statement as a definition of $\operatorname{dim} E$ ).

Let us fix a homomorphism of a Lie group $\widetilde{L}$ into the group of automorphisms of $A$. It generates a homomorphism of a Lie algebra $L$ into Lie algebra of derivations (infinitesimal automorphisms) of $A$. The derivation of $A$ corresponding to $X \in L$ will be denoted by $\delta_{X}$; derivations corresponding to the elements of a basis of $L$ will be denoted by $\delta_{1}, \ldots, \delta_{n}$.

Using the Lie algebra $L$ we can define a connection in an $A$-module $E$ as a set of linear operators $\nabla_{1}, \ldots, \nabla_{n}$ acting on $E$ and obeying the Leibnitz rule:

$$
\nabla_{\alpha}(\xi a)=\left(\nabla_{\alpha} \xi\right) a+\xi \delta_{\alpha} a .
$$

Sometimes it is convenient to define connection by means of operators $\nabla_{X}$ that depend linearly on $X \in L$ and obey

$$
\nabla_{X}(\xi a)=\left(\nabla_{X} \xi\right) a+\xi \delta_{X} a
$$

We will always consider Hermitian connections (i.e. the operator $\nabla_{\alpha}$ should be antiHermitian with respect to inner product in $E:\left\langle\nabla_{\alpha} \xi, \eta\right\rangle+\left\langle\xi, \nabla_{\alpha} \eta\right\rangle=$ $\left.\delta_{\alpha}<\xi, \eta>\right)$.

The curvature $F$ of connection is a 2 -form

$$
F_{X Y}=\left[\nabla_{X}, \nabla_{Y}\right]-\nabla_{[X, Y]} .
$$

This form is defined on the Lie algebra $L$ and takes values in $\operatorname{End}_{A} E$.

To work with connections in rigorous way one should consider instead of the algebra $A$ its dense subset $A^{\infty}$ consisting of elements that are smooth with respect to the action of the Lie group $\widetilde{L}$ on $A$. In similar way one should replace an $A$-module $E$ with its "smooth part" $E^{\infty}$.

\section{$3 \quad$ Noncommutative tori.}

By definition, an $n$-dimensional noncommutative torus is an associative algebra with involution having unitary generators $U_{1}, \ldots, U_{n}$ obeying 


$$
U_{k} U_{j}=e^{2 \pi i \theta_{k j}} U_{j} U_{k}
$$

Notice that different matrices $\theta_{j k}$ can determine isomorphic algebras; in particular replacing $\theta_{j k}$ by $\theta_{j k}+n_{j k}$ where $n_{j k} \in \mathbb{Z}$, we obtain the same commutation relations and therefore the same noncommutative torus. In two-dimensional case we consider $\theta$ in the notation $T_{\theta}$ as a number (two-dimensional antisymmetric matrix is determined by one number $\theta=\theta_{12}$, therefore we can identify the matrix with this number).

One can check that the numbers $\theta$ and $\widehat{\theta}$ determine Morita equivalent twodimensional tori if

$$
\widehat{\theta}=(A \theta+B)(C \theta+D)^{-1}
$$

where $A, B, C, D$ are integers and the matrix

$$
\left(\begin{array}{ll}
A & B \\
C & D
\end{array}\right)
$$

has determinant 1. Similar statement is correct for multidimensional tori [3], however in this case one should assume that $A, B, C, D$ are $n \times n$ matrices with integer entries obeying

$$
A^{t} C+C^{t} A=B^{t} D+D^{t} B=0, A^{t} D+C^{t} B=1
$$

where ${ }^{t}$ denotes transpose.

The noncommutative torus $T_{\theta}$ can be considered as an algebra of formal expressions

where $X$ runs over a lattice $D \subset \mathbb{R}^{n}$,

$$
\sum C_{X} U_{X}
$$

$$
U_{X} U_{Y}=e^{\pi i \vartheta_{X Y}} U_{X+Y}
$$

$\vartheta_{X Y}$ is a bilinear form on $D$ and $U_{X}^{*}=U_{X}^{-1}=U_{-X}$. (By definition a discrete subgroup $D$ of vector space $V$ is a lattice if $V / D$ is compact.) It is convenient to assume that the coefficients $C_{X}$ belong to the Schwartz space $\mathcal{S}$, i.e. that they vanish at infinity faster than any power. This assumption corresponds to consideration of the "smooth part" $T_{\theta}^{\infty}$ of the torus $T_{\theta}$. It is easy to check that

$$
U_{X} U_{Y}=e^{2 \pi i \theta_{X Y}} U_{Y} U_{X}
$$

where $\theta_{X Y}=\left(\vartheta_{X Y}-\vartheta_{Y X}\right) / 2$; therefore operators $U_{j}=U_{e_{j}}$ where $e_{1}, \ldots, e_{n}$ is a lattice basis obey (8) with $\theta_{j k}=\theta_{e_{j}, e_{k}}$; this remark relates the new description of $T_{\theta}$ with the old one.

If we represent an element of $T_{\theta}$ by means of a complex-valued function $C_{X}$ on the lattice $D$ then the multiplication in $T_{\theta}$ can be specified by the formula

$$
\left(C * C^{\prime}\right)_{X}=\sum_{Y \in D} e^{\pi i \vartheta_{Y, X-Y}} C_{Y} C_{X-Y}^{\prime} .
$$


Instead of $C_{X}$ we can consider its "Fourier transform"-a function on the torus $\left(\mathbb{R}^{n}\right)^{*} / D^{*}$ defined as

$$
f(\xi)=\sum_{X \in D} C_{X} e^{2 \pi i \xi X}
$$

Here $D^{*}$ is a lattice dual to $D$. It consists of such points $\xi \in\left(\mathbb{R}^{n}\right)^{*}$ that $\xi X$ is an integer for all $X \in D$. We can regard noncommutative torus $T_{\theta}$ as quantum deformation of commutative torus $\left(\mathbb{R}^{n}\right)^{*} / D^{*}$. The multiplication of functions $f, f^{\prime}$ on $\left(\mathbb{R}^{n}\right)^{*} / D^{*}$, considered as elements of $T_{\theta}$, is given by the formula:

$$
\left(f * f^{\prime}\right)(\xi)=\left(e^{\pi i \theta_{\alpha \beta} \frac{\partial}{\partial \xi_{\alpha}} \frac{\partial}{\partial \xi_{\beta}^{\prime}}} f(\xi) f^{\prime}\left(\xi^{\prime}\right)\right)_{\xi=\xi^{\prime}}
$$

Corresponding commutator can be identified with Moyal bracket.

One can define a trace on the algebra $T_{\theta}$ assigning to an element, represented by a function $C_{X}$ on the lattice, the value of this function at $X=0$. The trace, defined by means of this construction is unique ( up to a factor).

To construct a module over $T_{\theta}$ we should find a Hilbert space $\mathcal{H}$ and unitary operators $U_{X}$ on $\mathcal{H}$ obeying (14). Let us work with right modules and use the notation $\xi U_{X}$ where $\xi \in \mathcal{H}$. To introduce a structure of $C^{*}$-module in $\mathcal{H}$ we should define a $T_{\theta}$-valued inner product $<,>$ starting with $\mathbb{C}$-valued inner product $($,$) . This can be done by means of the formula$

$$
<\xi, \eta>=\sum_{X \in D}\left(\xi, \eta U_{-X}\right) U_{X} .
$$

Under certain assumptions about convergence of the series in (18) it is easy to check that the inner product $\langle$,$\rangle satisfies the conditions in the definition$ of $C^{*}$-module. It is more difficult to formulate easily verifiable conditions of projectivity of the module. ( The most convenient way to prove projectivity is to check that the unit operator can be represented as an endomorphism of finite rank, i.e. as an operator defined by the formula $\left.x \rightarrow \sum<\xi_{i}, x>\eta_{i}\right)$.

The situation in the case of left modules is the same. (Every left $T_{\theta}$-module can be considered as a right $T_{-\theta}$-module.)

Examples of $T_{\theta}$-modules can be obtained in the following way. Let us define operators $U_{\gamma, \widetilde{\gamma}}$ in the space $E=L^{2}\left(\mathbb{R}^{n}\right)$ by the formula:

$$
\left(U_{\gamma, \tilde{\gamma}} f\right)(X)=e^{2 \pi i \tilde{\gamma} X} f(X+\gamma) .
$$

Here $\gamma \in \mathbb{R}^{n}, \widetilde{\gamma} \in\left(\mathbb{R}^{n}\right)^{*}$. It easy to check that

$$
U_{\gamma, \tilde{\gamma}} U_{\lambda, \tilde{\lambda}}=e^{2 \pi i \tilde{\gamma} \lambda} U_{\gamma+\lambda, \tilde{\gamma}+\tilde{\lambda}}
$$

Using this relation one can construct a left $T_{\theta}$-module $E_{\Gamma}$ by means of operators $U_{\gamma, \widetilde{\gamma}}$ where $(\gamma, \widetilde{\gamma}) \in \Gamma \subset V=\mathbb{R}^{n}+\left(\mathbb{R}^{n}\right)^{*}$ and $\Gamma$ is a lattice in $V$. (The requirement that $\Gamma$ be a lattice is necessary to prove that $E_{\Gamma}$ is a projective module.) It follows from $(20)$ that the operators $U_{\gamma, \tilde{\gamma}}, U_{\lambda, \tilde{\lambda}}$, where $(\gamma, \widetilde{\gamma}),(\lambda, \widetilde{\lambda}) \in \Gamma$ 
obey (14) with $\theta_{(\gamma, \widetilde{\gamma}),(\lambda, \widetilde{\lambda})}=\widetilde{\gamma} \lambda-\widetilde{\lambda} \gamma$, and therefore specify a module over noncommutative torus. ( More precisely, to define $T_{\theta}$-valued inner product we should restrict the class of functions taking only functions from the Schwartz class $S\left(\mathbb{R}^{n}\right)$.)

Let us define the lattice $\Gamma^{*}$ as a group of all $(\mu, \widetilde{\mu}) \in \mathbb{R}^{n}+\left(\mathbb{R}^{n}\right)^{*}$ obeying the condition

$$
\widetilde{\mu} \gamma-\widetilde{\gamma} \mu \in \mathbb{Z}
$$

It is easy to check that every operator $U_{\mu, \tilde{\mu}}$ where $(\mu, \widetilde{\mu}) \in \Gamma^{*}$ commutes with all operators $U_{\gamma, \widetilde{\gamma}}$ where $(\gamma, \widetilde{\gamma}) \in \Gamma$. One can prove that every endomorphism of $E_{\Gamma}$ belongs to the closure ( for the $C^{*}$-norm ) of the linear span of $U_{\mu, \tilde{\mu}}$ where $(\mu, \widetilde{\mu}) \in \Gamma^{*}$. This means that $\operatorname{End}_{T_{\theta}} E_{\Gamma}$ can be identified with the torus $T_{\widehat{\theta}}$, where $\widehat{\theta}_{(\mu, \widetilde{\mu})(\nu, \widetilde{\nu})}=-\widetilde{\mu} \nu+\widetilde{\nu} \mu$ is a bilinear form on $\Gamma^{*}$. The torus $T_{\widehat{\theta}}$ is Morita equivalent to $T_{\theta}$. The space $S\left(\mathbb{R}^{n}\right)$ considered as a left $T_{\theta}$-mobile $E_{\Gamma}$ and a right $T_{\widehat{\theta}}$-mobile $E_{\Gamma^{*}}$ is a $\left(T_{\theta}, T_{\widehat{\theta}}\right)$-equivalence bimodule; we will denote this bimodule by $P_{\Gamma, \Gamma^{*}}$.

The above construction can be generalized in the following way. Let us consider instead of $\mathbb{R}^{n}$ any abelian group $G$ that can be represented as a direct sum of $\mathbb{R}^{n}$ and finitely generated abelian group $G^{\prime}$ (in other words $G^{\prime}$ is a direct sum of several copies of $\mathbb{Z}$ and finite cyclic groups $\left.\mathbb{Z}_{m}\right)$. Then we can consider operators $\left(U_{\gamma, \widetilde{\gamma}} f\right)(x)=e^{2 \pi i \widetilde{\gamma}(x)} f(x+\gamma)$ where $x \in G, \gamma \in G, \widetilde{\gamma} \in G^{*}$. Here $G^{*}$ stand for the group of characters of $G$ (i.e. a group of continuous homomorphisms of $G$ into the group $K=\mathbb{R} / \mathbb{Z}$ ).

Every lattice $\Gamma \subset G \times G^{*}$ determines a module over noncommutative torus. Endomorphisms of this module can be described by means of dual lattice $\Gamma^{*}$, that consists of elements $(\mu, \widetilde{\mu}) \in G \times G^{*}$ obeying the condition

$$
\tilde{\mu} \gamma-\widetilde{\gamma} \mu=0
$$

for all $(\gamma, \widetilde{\gamma}) \in \Gamma$. (Notice that $\widetilde{\mu} \gamma=\widetilde{\mu}(\gamma)$ and $\widetilde{\gamma} \mu=\widetilde{\gamma}(\mu)$ are considered as elements of $K=\mathbb{R} / \mathbb{Z}$ ).

In particular, for two-dimensional noncommutative torus $T_{\theta}$ we can take $G=\mathbb{R} \times \mathbb{Z}_{q}$ and a lattice $\Gamma_{p, q}$ spanned by elements $\left(\gamma_{1}, \widetilde{\gamma}_{1}\right),\left(\gamma_{2}, \widetilde{\gamma}_{2}\right)$ where $\gamma_{1}=(0,-p), \gamma_{2}=(1,0), \widetilde{\gamma}_{1}=(p / q-\theta, 0), \widetilde{\gamma}_{2}=(0,-1)$. (We consider $\mathbb{Z}_{q}$ as $\mathbb{Z} / q Z$ and identify $G^{*}$ with $\mathbb{R} \times \mathbb{Z}_{q}$.) Corresponding $T_{\theta}$-module will be denoted by $E_{p, q}$. (Here $p \in \mathbb{Z}, q \in \mathbb{Z}$.)

Let us consider as an example the case when the lattice $\Gamma \subset \mathbb{R}^{2}$ is spanned by the vectors $(1,0),(0, \theta+n)$ where $n \in \mathbb{Z}, \theta \in \mathbb{R}$. The lattice $\Gamma^{*}$ is spanned by vectors $(1,0)$ and $\left(0,(\theta+n)^{-1}\right)$. We obtain that two-dimensional tori $T_{\theta}$ and $T_{(\theta+n)^{-1}}$ are Morita equivalent. (Recall that the tori $T_{\theta}$ and $T_{\theta+n}$ coincide.) We will analyze the relation between $T_{\theta}$-modules and $T_{(\theta+n)^{-1}}$-modules that stems from Morita equivalence between $T_{\theta}$ and $T_{(\theta+n)^{-1}}$.

To every pair $(p, q)$ of integers we assigned a $T_{\theta}$-module $E_{p, q} ;$ if $\theta$ is irrational we obtain a one-to one correspondence between pairs $(p, q) \in \mathbb{Z}^{2}$ obeying $p-q \theta>$ 0 and (classes of projective) modules. It follows from our general results (see 


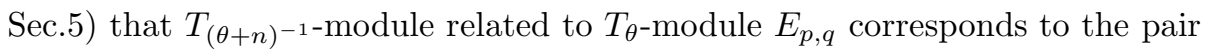
$(-q,-(p+q n))$.

We will give now a direct proof of this result for the case of the module $E_{p, 1}$. Such a module can be described as a right module $E_{\Gamma}$ corresponding to a lattice $\Gamma_{p, 1}$ spanned by $(1,0),(0, \theta+p)$. The tensor product $E_{\Gamma_{p, 1}} \otimes_{\mathbb{C}} P_{\Gamma, \Gamma^{*}}$ can be regarded as a space of functions $f(x, y)$ depending on two variables should identify $f(x+1, y)$ with $f(x, y+1)$ and $x \in \mathbb{R}, y \in \mathbb{R}$. To obtain $\widehat{E}=E_{\Gamma_{p, 1}} \otimes_{T_{\theta}} P_{\Gamma, \Gamma^{*}}$ wee $e^{-2 \pi i(\theta+p) x} f(x, y)$ with $e^{2 \pi i(\theta+n) y} f(x, y)$. It is easier to describe the dual space $\widehat{E}^{*}$ that consists of generalized functions $g(x, y)$ obeying

$$
\begin{gathered}
g(x+1, y)=g(x, y+1) \\
e^{-2 \pi i(\theta+p) x} g(x, y)=e^{2 \pi i(\theta+n) y} g(x, y)
\end{gathered}
$$

One can check that an element of $\widehat{E}^{*}$ (a solution to $\left.(23),(24)\right)$ can be represented in the form

$$
g(x, y)=\sum_{k} \delta(\theta(x+y)+p x+n y-k) g_{k}(x+y)
$$

where $g_{k}(x)=g_{k+(p+n)}(x)$. In other words we can identify elements of $(\widehat{E})^{*}$ with functions on $\mathbb{R} \times \mathbb{Z}_{p+n}$. By definition the action of $T_{(\theta+n)^{-1}}$ on $\widehat{E}$ is induced by the action of $T_{(\theta+n)^{-1}}$ on $P_{\Gamma, \Gamma^{*}}$. This means that we should consider operators on $E \otimes_{\mathbb{C}} P_{\Gamma, \Gamma^{*}}$ that transform $f(x, y)$ into $f(x, y+1)$ and $e^{2 \pi i(\theta+n)^{-1} y} f(x, y)$ respectively. These operators induce operators on $\widehat{E}$ and on $(\widehat{E})^{*}$. It is easy to describe explicitly the induced operators on $(\widehat{E})^{*}$. It follows from this descrip-

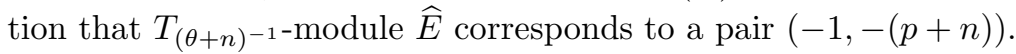

\section{Compactifications of M(atrix) theory.}

Let us consider the case when the $C^{*}$-algebra $A$ has a trace $\operatorname{Tr}$ and $L$ is a ten-dimensional commutative Lie algebra, equipped with inner product $($,$) .$

Let us fix an orthonormal basis in $L$ with respect to this inner product. Then for every $A$-module $E$ we can define a functional $I$ by the formula

$$
I=\sum_{\alpha, \beta} \operatorname{Tr} F_{\alpha \beta}^{2}+2 \sum \operatorname{Tr} \Psi^{i} \Gamma_{i j}^{\alpha}\left[\nabla_{\alpha}, \Psi^{j}\right]
$$

Here $F_{\alpha \beta}$ are components of the curvature tensor of connection $\nabla_{\alpha}$ with respect to the orthonormal basis in $L$ and $\Psi^{i}, i=1, \ldots, 16$ are elements of $\Pi \operatorname{End}_{A} E$, where $\Pi$ stands for parity reversing. The symbol $\Gamma_{i j}^{\alpha}$ denotes tendimensional Dirac matrices.

We consider $I$ as a functional on Conn $\times\left(\Pi\right.$ End $\left.{ }_{A} E \otimes S\right)$ where Conn stands for the space of all Hermitian connections on $E$ and $S$ is the space of Weyl spinors. 
In the case when $A$ is the algebra of complex numbers, $E=\mathbb{C}^{N}$ is an $N$ dimensional vector space (free module) and the algebra $L$ acts trivially on $\mathbb{C}$ one can identify (26) with the action functional of IKKT matrix model.

In the case when $A$ is a noncommutative torus one can consider (26) as an action functional of toroidal compactification of IKKT M(atrix) theory; this fact follows from the results of [1]. To verify this statement we notice that the relations (8) remain correct if we replace $U_{k}$ with $\widetilde{U}_{k}=\lambda_{k} U_{k}$ where $\left|\lambda_{k}\right|=1$. This means that we can consider an $n$-dimensional Lie algebra $L_{\theta}$ of derivations of $T_{\theta}$; the generators of $L_{\theta}$ act by the formula $\delta_{j} U_{k}=i U_{k}$ if $j=k, \delta_{j} U_{k}=0$ if $j \neq k$. An action of ten-dimensional commutative algebra $L$ on $T_{\theta}$ is defined by means of an arbitrary surjective linear map of $L=R^{10}$ onto $L_{\theta}=R^{n}$. Corresponding connections can be identified with the solutions to Eqn (3.14) of $[1]$.

One can modify the functional (26) replacing $F_{\alpha \beta}$ with $F_{\alpha \beta}+\varphi_{\alpha \beta} \cdot 1$ where $\varphi_{\alpha \beta}$ is an antisymmetric 2 -form on $L$. We obtain a functional

$$
J=\sum_{\alpha, \beta} \operatorname{Tr}\left(F_{\alpha \beta}+\varphi_{\alpha \beta} \cdot 1\right)^{2}+2 \sum \operatorname{Tr} \Psi^{i} \Gamma_{i j}^{\alpha}\left[\nabla_{\alpha}, \Psi^{j}\right]
$$

that also was considered in [1].

The functional (27) depends on the following data (in the case $A=T_{\theta}$ ).

a) symmetric bilinear form $g_{\alpha \beta}$ on $L=R^{10}$ that determines an inner product on $L$ (we work in orthonormal basis, therefore $g_{\alpha \beta}=\delta_{\alpha \beta}$ );

b) antisymmetric bilinear form $\varphi_{\alpha \beta}$ on $L=R^{10}$;

c) antisymmetric bilinear form $\theta_{\alpha \beta}$ on $R^{n}$, specifying the algebra $T_{\theta}$;

d) surjective map $\delta: L=R^{10} \rightarrow L_{\theta}=R^{n}$;

e) $T_{\theta}$-module $E$.

We will show that using Morita equivalence we can transform this set of data into another set of data giving a physically equivalent functional (27).

Our starting point will be an $(A, \hat{A})$-equivalence bimodule $P$, i.e. a bimodule that gives Morita equivalence between $A=T_{\theta}$ and $\hat{A}=T_{\widehat{\theta}}$. (Our consideration is valid not only for tori, therefore we prefer to use more general notations.) We will assume that there exists a constant curvature connection $\nabla_{X}^{P}$ on $P$, considered as a left $A$-module. In other words, we require that

$$
\begin{gathered}
\nabla_{X}^{P}(a \xi)=a \nabla_{X}^{P} \xi+\left(\delta_{X} a\right) \cdot \xi \\
{\left[\nabla_{X}^{P}, \nabla_{Y}^{P}\right]=\sigma_{X Y} \cdot 1}
\end{gathered}
$$

Here $\xi \in P, a \in A, X \in L, \delta_{X}$ stands for the action of $L$ on $A, \sigma_{X Y}$ is an antisymmetric bilinear form on $L$. We impose a condition

$$
\nabla_{X}^{P}(\xi \hat{a})=\left(\nabla_{X}^{P} \xi\right) \hat{a}+\xi \widehat{\delta}_{X} \hat{a}
$$

where $\xi \in P, \hat{a} \in \hat{A}$. This means that $\nabla_{X}^{P}$ is also a connection in the right $\hat{A}$-module $P$ for an appropriate definition of an action $\widehat{\delta}_{X}$ of $L$ on $\hat{A}$. (Talking 
about an action of commutative Lie algebra $L$ on $A=T_{\theta}$ and on $\widehat{A}=T_{\widehat{\theta}}$ we will have in mind homomorphisms $\delta$ and $\widehat{\delta}$ from $L$ into $L_{\theta}$ and $L_{\widehat{\theta}}$ respectively; we assume that these homomorphisms are surjective.)

We will say that the $(A, \hat{A})$-module $P$ satisfying the conditions above determines complete Morita equivalence between $A$ and $\hat{A}$. It is easy to check that the bimodule $P_{\Gamma, \Gamma^{*}}$ constructed above determines complete Morita equivalence between $T_{\theta}$ and $T_{\widehat{\theta}}$.

Recall, that using the $(A, \hat{A})$-module $P$ we can construct a one-to-one correspondence between right $A$-modules and right $\hat{A}$-modules by the formula $\hat{E}=E \otimes_{A} P$.

If $\nabla_{X}$ is a connection in the right $A$-module $E$ (i.e. it obeys (6)) we can consider $\hat{\nabla}_{X}=\nabla_{X} \otimes 1+1 \otimes \nabla_{X}^{P}$ as on operator on $\hat{E}$. (One can obtain $\hat{E}=E \otimes_{A} P$ from $E \otimes_{\mathbb{C}} P$ by means of identification $\xi a \otimes \eta \sim \xi \otimes a \eta$. It is clear that $\widehat{\nabla}_{X}$ acts on $E \otimes_{\mathbb{C}} P$; to verify that $\widehat{\nabla}_{X}$ acts on $\hat{E}$ we should check the compatibility with this identification.)

Using (30) we check, that $\widehat{\nabla}_{X}$ is a connection in the right $\hat{A}$-module $\hat{E}$. Let us calculate its curvature $F_{X Y}^{\hat{\nabla}}=F_{X Y}^{\wedge}$. Notice that the curvature $F_{X Y}=F_{X Y}^{\nabla}$ of the connection $\nabla_{X}$ for fixed $X, Y \in L$ can be considered as an endomorphism of $E$; therefore we can construct the corresponding endomorphism $\widehat{F}_{X Y}$ of $\hat{E}$. It is easy to see that

$$
F_{X Y}^{\wedge}=\widehat{F}_{X Y}+\sigma_{X Y} \cdot 1
$$

This follows from the remark that considering $\widehat{\nabla}_{X}$ as an operator on $E \otimes_{\mathbb{C}} P$ we obtain

$$
\left[\widehat{\nabla}_{X}, \widehat{\nabla}_{Y}\right]=F_{X Y}+\sigma_{X Y} \cdot 1
$$

Both terms in the RHS of (32) induce operators on $\hat{E}=E \otimes_{A} P$; we immediately obtain (31) from (32) and the definition of $F_{X Y}^{\wedge}$. The formula (31) permits us to derive some important statements. We see first of all that in the case when the connection $\nabla_{X}$ has constant curvature the corresponding connection $\widehat{\nabla}_{X}$ also has constant curvature. (It equals to $\left(f_{X Y}+\sigma_{X Y}\right) \cdot 1$, where $f_{X Y}$ stands for the curvature of $\nabla_{X}$.) In other words, if $\nabla_{X}$ determines a BPS state having maximal supersymmetry, then $\hat{\nabla}_{X}$ has the same property. Analogous statement is true for BPS states having less supersymmetries. Recall that $\nabla_{X}$ determines a BPS state if one can find spinors $\varepsilon, \varepsilon^{\prime}$ obeying

$$
\varepsilon \Gamma^{\alpha \beta} F_{\alpha \beta}+\varepsilon^{\prime} \cdot 1=0
$$

It follows from (31) that then

$$
\varepsilon \Gamma^{\alpha \beta} F_{\alpha \beta}^{\wedge}+\varepsilon^{\prime \prime} \cdot 1=0
$$

where $\varepsilon^{\prime \prime}=\varepsilon^{\prime}-\varepsilon \Gamma^{\alpha \beta} \sigma_{\alpha \beta}$. We see that $\widehat{\nabla}_{X}$ determines a BPS state having the same number of supersymmetries as $\nabla_{X}$. 
Now we can represent the action functional $J$ defined on the space Conn $\times\left(\operatorname{End}_{A} E \otimes S\right)$ as an action functional $\widehat{J}$ defined on the space $\widehat{\operatorname{Conn}} \times\left(\operatorname{End}_{\hat{A}} \widehat{E} \otimes\right.$ $S$ ), where $\widehat{C o n n}$ stands for the space of connections on $\hat{A}$-module $\hat{E}$, that are defined by means of the action $\widehat{\delta}_{X}$ of $L$ on $\hat{A}$. Expressing $J$ in terms of connections $\widehat{\nabla}_{X}$ and endomorphisms $\widehat{\Psi}^{i}$ corresponding to $\nabla_{X}$ and $\Psi^{i}$ we obtain

$$
\widehat{J}=\sum_{\alpha, \beta} \operatorname{Tr}\left(\widehat{F}_{\alpha \beta}+\left(\varphi_{\alpha \beta}-\sigma_{\alpha \beta}\right) \cdot 1\right)^{2}+\sum \operatorname{Tr} \widehat{\Psi}^{i} \Gamma_{i j}^{\alpha}\left[\widehat{\nabla}_{\alpha}, \widehat{\Psi}^{j}\right]
$$

To be sure that the theory based on the consideration of the action functional $\widehat{J}$ is equivalent to the theory based on $J$ we should check that the correspondence Conn $\rightarrow \widehat{\text { Conn }}$ we constructed is bijective. The proof is based on the construction of the inverse map $\widehat{\text { Conn }} \rightarrow$ Conn. Recall that to prove bijectivity of map $E \rightarrow$ $\hat{E}=E \otimes_{A} P$ we used an $(\hat{A}, A)$-equivalence bimodule $\bar{P}$. Here we use the same bimodule equipped with a connection $\bar{\nabla}_{\alpha}$ defined by the formula

$$
\bar{\nabla}_{\alpha}(\bar{\omega})=\overline{\left(\nabla_{\alpha} \omega\right)}
$$

The fact, that $E=\hat{E} \otimes_{\hat{A}} \bar{P}$ follows from the remark that $P \otimes_{\hat{A}} \bar{P}=A$ as $(A, A)$ bimodule. To prove similar fact for connections we notice that the operator $\nabla_{\alpha} \otimes 1+1 \otimes \bar{\nabla}_{\alpha}$ on $P \otimes_{\mathbb{C}} \bar{P}$ induces operator $\delta_{\alpha}$ on $A=P \otimes_{\hat{A}} \bar{P}$. To check this we notice that the natural map of $P \otimes_{\mathbb{C}} \bar{P}$ onto $P \otimes_{\hat{A}} \bar{P}=A$ can be identified with inner product $\langle\cdot, \cdot\rangle_{A}$ in $P$. Then the statement we need follows immediately from the assumption that $\nabla_{\alpha}$ is a Hermitian connection.

\section{Detailed analysis of toroidal compactifications.}

Let us give a detailed analysis of the above constructions for the case when $A$ is a noncommutative torus $T_{\theta}$. It is shown in [3] that $T_{\widehat{\theta}}$ is Morita equivalent to $T_{\theta}$ if $\widehat{\theta}=g \theta$ where $g \in S O(n, n \mid \mathbb{Z})$, i.e. $g$ is a matrix of the form (10) having integral entries and unit determinant and obeying (11). (The definition of the action of $S O(n, n \mid \mathbb{Z})$ on a dense subset of the space of antisymmetric matrices is given by the formula(9) .) It is easy to check that the proof of Morita equivalence in [3] gives complete Morita equivalence $T_{\theta} \sim T_{g \theta}$ in the sense of present paper (the $\left(T_{\theta}, T_{g \theta}\right)$-equivalence bimodule constructed in [3] can be represented as a bimodule $P_{\Gamma, \Gamma^{*}}$ for appropriate choice of lattices $\Gamma, \Gamma^{*} \subset G \times G^{*}$ and therefore it can be equipped with a connection obeying the conditions we imposed). We will prove that, conversely, if the noncommutative $T_{\widehat{\theta}}$ is completely Morita equivalent to the torus $T_{\theta}$ then $\widehat{\theta}$ and $\theta$ belong to the same orbit of the group $S O(n, n \mid \mathbb{Z})$. In other words, we will see that $T_{\widehat{\theta}}$ is completely Morita equivalent to $T_{\theta}$ if and only if $\widehat{\theta}=g \theta$ for some $g \in S O(n, n \mid \mathbb{Z})$.

We will give also an explicit description of the correspondence between projective modules over $T_{\theta}$ and projective modules over $T_{\widehat{\theta}}$.

We will start with formulation of some well known results. Notice that the Lie algebra $L_{\theta}$ of derivations of $T_{\theta}$ can be considered as a Lie algebra of a 
Lie group $\widetilde{L}_{\theta}$ of automorphisms of $T_{\theta}$. The Lie group $\widetilde{L}_{\theta}$ is a torus that can be identified with $L_{\theta} / D$, where $D=\mathbb{Z}^{n}$ is a lattice in $L_{\theta}$. One can consider an element of $\widetilde{L}_{\theta}$ as a row $\left(\lambda_{1}, \ldots, \lambda_{n}\right)$ where $\left|\lambda_{i}\right|=1$; such a row acts on $T_{\theta}$ transforming $U_{j}$ into $\lambda_{j} U_{j}$, where $U_{j}$ are generators of $T_{\theta}$. Then $D$ can be regarded as a lattice generated by $e_{j}=2 \pi \delta_{j} \in L_{\theta}$ where $\delta_{j} U_{k}=i U_{k}$ for $j=k, \delta_{j} U_{k}=0$ for $j \neq k$. Let us denote by $\mathcal{F}$ the Grassmann algebra $\Lambda\left(L_{\theta}\right)$. An element of $\mathcal{F}$ can be considered as a formal linear combination

$$
\omega=\sum_{k} \sum_{j_{1} \ldots j_{k}} \omega^{j_{1} \ldots j_{k}} e_{j_{1}} \ldots e_{j_{k}}
$$

where $\omega^{j_{1} \ldots j_{k}} \in \mathbb{C}$ is antisymmetric with respect to $j_{1}, \ldots j_{k}$. Requiring that $\omega^{j_{1} \ldots j_{k}}$ in (36) be integers we single out a subring $\Lambda(D) \subset \Lambda\left(L_{\theta}\right)$. Similarly, using the lattice $D^{*} \subset L^{*}$ that is dual the lattice $D \subset L$ we define the "integral part" $\Lambda\left(D^{*}\right)$ of $\mathcal{F}^{*}=\Lambda\left(L^{*}\right)$. One can identify the group $K_{0}\left(T_{\theta}\right)$ with even part $\Lambda^{e v}\left(D^{*}\right)$ of $\Lambda\left(L^{*}\right)$ (the ring $\Lambda\left(D^{*}\right)$ inherits $\mathbb{Z}_{2}$-grading from $\Lambda\left(L_{\theta}^{*}\right)$; we define its even part with respect to this grading). Recall that to define $K_{0}(A)$ one should apply the Grothendieck construction to the semigroup of equivalence classes of projective $A$-modules with respect to direct sum. (In other words we consider formal differences $E_{1}-E_{2}$ where $E_{1}, E_{2}$ are projective $A$-modules and identify $\left(E_{1}-E_{2}\right)+E_{2}$ with $E_{1}$; see Appendix for more information about $K_{0}$.) In particular, every projective $A$-module $E$ determines an element $\mu(E) \in$ $K_{0}(A)$; the set $K_{0}^{+}(A)$ of elements of $K_{0}(A)$ that can be obtained this way is by definition the positive cone in $K_{0}(A)$. In the case $A=T_{\theta}$ and $\theta$ is irrational (i.e. at least one entry of the matrix $\theta$ is irrational) one can prove that two nonequivalent projective $A$-modules determine different elements of $K_{0}(E)$; this permits us to identify a projective $A$-module with its representative $\mu=\mu(E) \in$ $K_{0}^{+}(A)$.

The Chern character ch $E$ of a projective $A$-module $E$ is defined by the formula

$$
\operatorname{ch} E=\widehat{\tau}\left(e^{F / 2 \pi i}\right)=\sum_{n=0} \frac{1}{n !} \widehat{\tau}\left(F^{n}\right) \cdot \frac{1}{(2 \pi i)^{n}}
$$

where $F$ is a curvature of an arbitrary connection on $E$. Recall that to define a connection we fixed a homomorphism $\delta$ of a Lie algebra $L$ into the Lie algebra of derivations of $A$. Then the curvature $F$ can be considered as a two-form on $L$ taking values in the algebra $\widehat{A}=\operatorname{End}_{A} E$ and $F^{n}$ is a $2 n$-form taking values in $\widehat{A} \otimes \ldots \otimes \widehat{A}$. We assume that the algebra $A$ is equipped with a trace $\tau$; as we mentioned already then we can define a trace $\widehat{\tau}$ on $\widehat{A}$ and therefore on $\widehat{A} \otimes \ldots \otimes \widehat{A}$. The formula (37) determines the Chern character $\operatorname{ch}(E)$ as an inhomogeneous form on $L$ (i.e. as an element of the Grassmann algebra $\mathcal{F}^{*}=\Lambda\left(L^{*}\right)$ that is dual to $\mathcal{F}=\Lambda(L)$. (Notice that the definition of connection that we use is a particular case of more general definition that we do not need. In this general definition the Chern character takes values in cyclic homology of $A$.)

If $A$ is a noncommutative torus $T_{\theta}$ one can consider the matrix $\theta$ as a 2 -form 
on $L=L_{\theta}$, i.e. as an element of $\theta \in \Lambda(L)$. Then one can prove the formula :

$$
\left.\operatorname{ch} E=e^{\theta}\right\lrcorner \mu(E),
$$

where $\lrcorner$ stands for the operation of contraction. It is convenient to consider elements $\mathcal{F}^{*}=\Lambda\left(L^{*}\right)$ as functions of anticommuting variables $\alpha^{1}, \ldots, \alpha^{n}$. Then one can write the formula (38) in the form

$$
\operatorname{ch} E=e^{\frac{1}{2} b_{k} \theta^{k j} b_{j}} \mu(E)
$$

where $b_{j}$ stands for the derivative with respect anticommuting variable $\alpha^{j}$. One can consider $\mathcal{F}^{*}$ also as a fermionic Fock space where $b_{1}, \ldots, b_{n}$ play the role of annihilation operators, and operators $a^{i}$ defined by means of multiplication by $\alpha^{i}$ play the role of creation operators. (The operators $a^{i}, b_{i}$ satisfy canonical anticommutation relations $\left[b_{k}, a^{j}\right]_{+}=\delta_{k}^{j},\left[a^{j}, a^{k}\right]_{+}=\left[b_{j}, b_{k}\right]_{+}=0$

Let us fix complete Morita equivalence between $A=T_{\theta}$ and $\widehat{A}=T_{\widehat{\theta}}$. We will study the relation between $\mu=\mu(E)$ and $\widehat{\mu}=\mu(\widehat{E})$ where $\widehat{A}$-module $\widehat{E}$ corresponds to the $A$-module $E$. Recall, that for every connection $\nabla$ in $E$ we constructed a connection $\widehat{\nabla}$ in $\widehat{E}$ and that the curvatures of $\nabla$ and $\widehat{\nabla}$ are related by the formula (31). It follows from $(37),(31)$ that

$$
\begin{gathered}
\operatorname{ch} E=\widehat{\tau} \exp \left(\frac{1}{2 \pi i} \alpha^{k} F_{k j}^{\nabla} \alpha^{j}\right) \\
\operatorname{ch} \widehat{E}=\tau \exp \left(\frac{1}{2 \pi i} \alpha^{k} F_{k j}^{\widehat{\nabla}} \alpha^{l}\right)=e^{\frac{1}{2 \pi i} \alpha^{k} \varphi_{k j} \alpha^{l}} \operatorname{ch} E
\end{gathered}
$$

where we identified $L_{\theta}$ and $L_{\widehat{\theta}}$ with $\mathbb{R}^{n}$ in the way that was used in (31). Applying (39),(40),(41) we relate $\mu$ and $\widehat{\mu}$ in the following way:

$$
\widehat{\mu}=V_{1} V_{2} V_{3} V_{4} \mu
$$

where

$$
\begin{gathered}
V_{1} f=\exp \left(-\frac{1}{2} b_{k} \widehat{\widehat{\theta}}^{k j} b_{j}\right) f \\
V_{2} f=\exp \left(\frac{1}{2 \pi i} \alpha^{k} \varphi_{k j} \alpha^{j}\right) f=\exp \left(\frac{1}{2 \pi i} a^{k} \varphi_{k j} a^{j}\right) f \\
V_{3} f=f(A \alpha) \\
V_{4} f=\exp \left(\frac{1}{2} b_{k} \theta^{k j} b_{j}\right) f
\end{gathered}
$$

The operators $V_{1}$ and $V_{4}$ relate $\widehat{\mu}$ and $\operatorname{ch}(\widehat{E}), \mu$ and $\operatorname{ch}(E)$, the operator $V_{2}$ relates $\operatorname{ch}(\widehat{E})$ and $\operatorname{ch}(E)$ and the operator $V_{3}$ should be included to take into account that (31) is correct only for special identification of $L_{\theta}$ and $L_{\widehat{\theta}}$. It is easy to check that the operators $V_{k}$ can be considered as linear canonical transformations. Recall that the operator $V$ acting on the Fock space is a linear canonical transformation if

$$
V a^{k} V^{-1}=A_{j}^{k} a^{j}+B^{k j} b_{j}
$$




$$
V b_{k} V^{-1}=C_{k j} a^{j}+D_{k}^{j} b_{j}
$$

or in matrix notations

$$
\begin{aligned}
& V a V^{-1}=A a+B b \\
& V b V^{-1}=C a+D b
\end{aligned}
$$

It is easy to check that the $2 n \times 2 n$ matr ices

$$
\widetilde{V}=\left(\begin{array}{cc}
A & B \\
C & D
\end{array}\right)
$$

belong to the group $O(n, n \mid \mathbb{C})$ i.e. they obey (11). The matrix $\widetilde{V}$ determines the operator $V$ up to a constant factor, therefore the group of linear canonical transformations can be identified with $O(n, n \mid \mathbb{C}) \times \mathbb{C}^{*}$. It is easy to calculate that

$$
\widetilde{V}_{1}=\left(\begin{array}{ll}
1 & -\widehat{\theta} \\
0 & 1
\end{array}\right), \widetilde{V}_{2}=\left(\begin{array}{ll}
1 & 0 \\
\Phi & 1
\end{array}\right), \widetilde{V}_{3}=\left(\begin{array}{ll}
A & 0 \\
0 & \left(A^{t}\right)^{-1}
\end{array}\right), \widetilde{V}_{4}=\left(\begin{array}{ll}
1 & \theta \\
0 & 1
\end{array}\right)
$$

Here $\Phi$ stands for the matrix $(2 \pi i)^{-1} \varphi_{k j}$. All these matrices belong to $S O(n, n \mid \mathbb{R})$; hence their product belongs to the same group. The operator $W=V_{1} V_{2} V_{3} V_{4}$ transforms the integral lattice $\Lambda^{e v}\left(D^{*}\right)$ into itself; this means that $\widetilde{W}=\widetilde{V}_{1} \widetilde{V}_{2} \widetilde{V}_{3} \widetilde{V}_{4}$ belongs to $S O(n, n \mid \mathbb{Z})$. We see that

$$
\widetilde{W}=\left(\begin{array}{ll}
A-\widehat{\theta} \Phi A & A \theta-\widehat{\theta}\left(\Phi A \theta+\left(A^{t}\right)^{-1}\right) \\
\Phi A & \Phi A \theta+\left(A^{t}\right)^{-1}
\end{array}\right)=\left(\begin{array}{ll}
M & N \\
R & S
\end{array}\right)
$$

where $M, N, R, S$ are matrices with integral entries,

$$
M^{t} R+R^{t} M=N^{t} S+S^{t} N=0, M^{t} S+R^{t} N=1 .
$$

We conclude from (53) that

$$
\Phi A=R, A=\widehat{\theta} R+M,\left(A^{t}\right)^{-1}=S-R \theta
$$

One can use these formulas to relate $\theta$ and $\widehat{\theta}$ :

$$
(\widehat{\theta} R+\mu) \cdot\left(S^{t}+\theta R^{t}\right)=1 .
$$

Another relation between $\theta$ and $\widehat{\theta}$ is

$$
N=(M+\widehat{\theta} R) \theta-\widehat{\theta} S .
$$

Using (54) one can check that (56) and (57) are equivalent. We see that

$$
\widehat{\theta}=(-M \theta+N)(R \theta-S)^{-1}
$$

and therefore $\widehat{\theta}=g \theta$ where $g \in S O(n, n \mid \mathbb{Z})$. We obtain that complete Morita equivalence of noncommutative tori is always of the kind described in [3]. 
Let us consider a fibration having the space of irrational antisymmetric $n \times n$ matrices $\theta$ as a base and a group $K_{0}\left(T_{\theta}\right)$ as a fiber. It follows from the results we mentioned that the set of all modules over $T_{\theta}$ with irrational $\theta$ is embedded in total space of this fibration. Let us consider a curve in the base connecting $\theta$ with $g \theta$ where $g \in S O(n, n \mid \mathbb{Z})$.It is easy to check that the monodromy transformation corresponding to this curve connects physically equivalent modules. The proof is based on a remark that for any curve the monodromy can be considered as a linear canonical transformation if the group $K_{0}\left(T_{\theta}\right)$ is identified with a lattice in the Fock space $\mathcal{F}^{*}$.

\section{Examples.}

Let us illustrate the above calculation on the example when $n=2 k$ and $M=$ $S=0, N=R=1$. Then we get Morita equivalence of the tori $A_{\theta}$ and $A_{\theta^{-1}}$. The operator $W$ transforming $\mu$ into $\widehat{\mu}$ obeys $W a^{i} W^{-1}=b_{i}$ and $W b_{i} W^{-1}=$ $a^{i}$ (i.e. it interchanges creation and annihilation operators). If $\mu=\mu(E)=$ $\sum_{k} \sum_{i_{1} \ldots i_{k}} \mu_{i_{1} \ldots i_{k}} \alpha^{i_{1}} \ldots \alpha^{i_{k}}$ then $\widehat{\mu}=\mu(\widehat{E})=W \mu=\sum_{l} \sum(* \mu)_{j_{1} \ldots j_{l}} \alpha^{j_{1}} \ldots \alpha^{j_{l}}$. In the case $n=2$ the operator $W$ transforms $\mu=p+q \alpha^{1} \alpha^{2}$ into $\widehat{\mu}=-q-p \alpha^{1} \alpha^{2}$. Corresponding Chern characters are

$$
\begin{gathered}
\mathrm{ch}=e^{\theta b_{1} b_{2}} \mu=(p-q \theta)+q \alpha^{1} \alpha^{2} \\
\widehat{\mathrm{ch}}=e^{\theta^{-1} b_{1} b_{2}} \widehat{\mu}=\left(-q+p \theta^{-1}\right)+p \alpha^{1} \alpha^{2} .
\end{gathered}
$$

We see that dimensions of modules $E$ and $\widehat{E}$ are equal to $\operatorname{dim} E=p-q \theta$ and $\operatorname{dim} \widehat{E}=-q+p \theta^{-1}=\theta^{-1} \cdot \operatorname{dim} E$ correspondingly.

Every projective module over two-dimensional noncommutative torus can be equipped with a constant curvature connection. One can calculate its curvature $F$ using (37) and (58). We get

$$
\begin{aligned}
& F=2 \pi i \frac{q}{p-q \theta} \alpha^{1} \alpha^{2} \\
& \widehat{F}=2 \pi i \frac{p \theta}{p-q \theta} \alpha^{1} \alpha^{2}
\end{aligned}
$$

for modules $E$ and $\widehat{E}$ respectively. Using (55) we see that $A=\theta^{-1}, \Phi=\theta$. Hence if $F^{\prime}$ is obtained from $F$ by means of the change of variables $\widetilde{\alpha}=A \cdot \alpha=\theta^{-1} \cdot \alpha$ we should have $F^{\prime}+2 \pi i \cdot \theta=\widehat{F}$. This is true:

$$
\frac{q}{p-q \theta} \theta^{2}+\theta=\frac{p \theta}{p-q \theta}
$$

In the case $n=4$ the operator $W$ transforms $\mu=p+\frac{1}{2} q_{i j} \alpha^{i} \alpha^{j}+s \alpha^{1} \alpha^{2} \alpha^{3} \alpha^{4}$ into $\widehat{\mu}=s+\frac{1}{2}(* q)_{i j} \alpha^{i} \alpha^{j}+p \alpha^{1} \alpha^{2} \alpha^{3} \alpha^{4}$. Corresponding Chern characters are

$$
\operatorname{ch}=p+\frac{1}{2} \operatorname{tr} \theta q+s \underline{\theta}+\frac{1}{2}(q+* \theta s)_{i j} \alpha^{i} \alpha^{j}+s \alpha^{1} \alpha^{2} \alpha^{3} \alpha^{4}
$$




$$
\widehat{\mathrm{ch}}=s+\frac{1}{2} \operatorname{tr} \theta^{-1}(* q)+p \underline{\left(\theta^{-1}\right)}+\frac{1}{2}\left(* q+*\left(\theta^{-1}\right) p\right)_{i j} \alpha^{i} \alpha^{j}+p \alpha^{1} \alpha^{2} \alpha^{3} \alpha^{4}
$$

Here $\underline{\theta}$ stands for the Pfaffian of $\theta$ (i.e. $\left.\underline{\theta}^{2}=\operatorname{det} \theta_{i j}\right),{\underline{\left(\theta^{-1}\right)}}^{\prime}=(\theta)^{-1}$. We obtain from $(64),(65)$ that $\operatorname{dim} \widehat{E}=(\operatorname{dim} E) \cdot \underline{\theta}^{-1}$. If there exists a constant curvature connection in $E$, then there exists such a connection in $\widehat{E}$ and corresponding curvatures up to a factor $2 \pi i$ can be identified with matrices

$$
F=\frac{q+(* \theta) s}{\operatorname{dim} E}, \widehat{F}=\frac{* q+*\left(\theta^{-1}\right) p}{\operatorname{dim} \widehat{E}}
$$

Taking into account that $A=\theta^{-1}, \Phi=\theta$ we can represent the relation (31) in the form

$$
-\theta F \theta+\theta=\widehat{F}
$$

One can check (67) directly using a non-trivial identify

$$
-\theta q \theta+\frac{1}{2} \theta \operatorname{Tr} \theta q=* q \underline{\theta}
$$

that is valid for all antisymmetric $4 \times 4$ matrices $\theta, q$.

\section{Relation to BFSS M(atrix) model.}

Let us study more thoroughly the action functional (27) in the case when there exists a constant curvature connection $\nabla_{\alpha}$ in $A$-module $E$. Then any other connection can be represented as $\nabla_{\alpha}+X_{\alpha}$ where $X_{\alpha} \in \operatorname{End}_{A} E$ and its curvature $F_{\alpha \beta}$ can be written in the form $F_{\alpha \beta}=f_{\alpha \beta} \cdot 1+\widetilde{\delta}_{\alpha} X_{\beta}-\widetilde{\delta}_{\beta} X_{\alpha}+\left[X_{\alpha}, X_{\beta}\right]$. where $f_{\alpha \beta}$ stands for the curvature of $\nabla_{\alpha}$ and $\widetilde{\delta}_{\alpha} X$ is defined as $\left[\nabla_{\alpha}, X\right]$. We can consider $(27)$ as a functional depending on $X_{\alpha} \in \operatorname{End}_{A} E, \alpha=1, \ldots, 10$, and $\Psi^{i} \in \Pi_{\operatorname{End}} E, i=1, \ldots, 16$; it is equal to

$J=\sum \operatorname{Tr}\left(\widetilde{\delta}_{\alpha} X_{\beta}-\widetilde{\delta}_{\beta} X_{\alpha}+\left[X_{\alpha}, X_{\beta}\right]+\left(f_{\alpha \beta}+\varphi_{\alpha \beta}\right) \cdot 1\right)^{2}+2 \sum \operatorname{Tr} \Psi^{i} \Gamma_{i j}^{\alpha}\left(\widetilde{\delta}_{\alpha} \Psi^{j}+\left[X_{\alpha}, \Psi^{j}\right]\right)$.

We used orthonormal coordinate system in $L$ in the expression for $J$. It is easy to rewrite this expression in arbitrary coordinate system by means of relations $X_{\alpha}=e_{\alpha}^{a} X_{a}, X_{a}=e_{a}^{\alpha} X_{\alpha}, \widetilde{\delta}_{\alpha}=e_{\alpha}^{a} \widetilde{\delta}_{a} \widetilde{\delta}_{a}=e_{a}^{\alpha} \widetilde{\delta}_{\alpha}$. In the case when End $_{A} E$ can be identified with $n$-dimensional noncommutative torus $T_{\rho}$ we can consider elements of $\operatorname{End}_{A} E$ as functions on (commutative) torus $L_{\rho}^{*} / D^{*}$; then for appropriate choice of coordinate systems on $L$ and on $L_{\rho}$ we can identify $\widetilde{\delta}_{a}$ for $1 \leq a \leq n$ with partial derivatives $\frac{\partial}{\partial \sigma^{a}}$ and assume that $\widetilde{\delta}_{a}=0$ for $a>n$. (We denote coordinates on $L_{\rho}^{*} / D^{*}$ by $\sigma^{1}, \ldots, \sigma^{n}$.) The same is correct if $\operatorname{End}_{A} E$ is an algebra of $N \times N$ matrices with entries from $T_{\rho}$. Similar result can be proved also in general case. ( It follows from well known results that for modules considered in Sec. $3 \operatorname{End}_{A} E$ can be represented as an algebra of functions on finitely generated abelian group with multiplication rule (15) where 
$\vartheta_{X Y}$ is a bicharacter.) Let us write down the expression for the functional $J$ in the latter case restricting ourselves to the bosonic part of $J$ for simplicity.

$$
\begin{gathered}
J_{b o s}=\sum_{a, b} \operatorname{Tr} F_{a b} F^{a b} \\
F_{a b}=\frac{\partial}{\partial \sigma^{a}} X_{b}-\frac{\partial}{\partial \sigma^{b}} X_{a}+\left[X_{a}, X_{b}\right]+\left(f_{a b}+\varphi_{a b}\right) \cdot 1 \text { for } 1 \leq a, b \leq n, \\
F_{a b}=\frac{\partial}{\partial \sigma^{a}} X_{b}+\left[X_{a}, X_{b}\right]+\left(f_{a b}+\varphi_{a b}\right) \cdot 1 \text { for } 1 \leq a \leq n, b>n, \\
F_{a b}=\left[X_{a}, X_{b}\right]+\left(f_{a b}+\varphi_{a b}\right) \cdot 1 \text { for } a>n, b>n .
\end{gathered}
$$

Tr stands for composition of matrix trace tr and integration over $\sigma^{1}, \ldots, \sigma^{n}$ (i.e. $\left.\operatorname{Tr} R=\int_{L_{\rho} / D} \operatorname{tr} R\left(\sigma^{1}, \ldots, \sigma^{n}\right) d^{n} \sigma\right)$. In (70) we raise indices by means of metric tensor $g_{a b}=e_{a}^{\alpha} e_{b}^{\alpha}$. We can replace this Euclidean metric tensor with metric tensor having Lorentzian signature (i.e. perform "Wick rotation") . This corresponds to consideraton of compactification of BFSS M(atrix) model.

We have proven that Morita equivalence gives us equivalence between toroidal compactifications of IKKT M(atrix) model. It is clear that performing corresponding Wick rotations we obtain equivalent compactifications of BFSS model. We will analyze this equivalence thoroughly in a forthcoming paper [10].

\section{APPENDIX}

Let us start with the following general observation about supermatrix models. We consider an action functional of the form

$$
S=\sum a_{\alpha_{1} \ldots \alpha_{n}} \operatorname{Tr} X^{\alpha_{1}} \ldots X^{\alpha_{n}}
$$

defined on a set of matrices $X^{\alpha}, 1 \leq \alpha \leq c$. We consider $X^{\alpha}$ as matrices of (even or odd) Hermitian operators acting in the space $\mathbf{C}^{K \mid L}$; in other words they are $(K \mid L) \times(K \mid L)$-matrices. Corresponding partition function $Z$ is defined by means of integration of $\exp (-S)$ over all Hermitian matrices. Then it is easy to check that $Z$ depends only on the difference $N=K-L$. One of the ways to prove this fact is to apply the standard representation of $Z$ in terms of a sum over all ribbon graphs. The size of the matrix enters in this representation only as $\operatorname{Tr} 1=K-L$.

Now we can consider toroidal compactifications of IKKT or BFSS matrix models taking into account the above remark. It was shown in [1] that such compactifications are related to connections in projective modules over commutative or non-commutative torus. More precisely, the equations arising in compactification problem cannot be solved by means of finite-dimensional matrices, but can be solved in terms of Hermitian operators in Hilbert spaces with action of (noncommutative) torus. These infinite-dimensional solutions can be used to obtain approximate finite-dimensional solutions. Now we see that we can work with the same success in $\mathbf{Z}_{2}$-graded Hilbert spaces. Then the approximate finite-dimensional solutions are supermatrices, but we have seen that the 
matrix model based on $(K \mid L) \times(K \mid L)$ supermatrices is equivalent to theory based on $(K-L) \times(K-L)$ matrices.

Let us remark now that the theory of $\mathbf{Z}_{2}$-graded projective modules is closely related to $K$-theory. The group $K_{0}(A)$ where $A$ is an associative algebra, can be define as a group of $\mathbf{Z}_{2}$-graded projective modules with equivalence relation $E \oplus \Pi E \backsim 0$. (Here $\Pi$ stand for parity reversion. The operation in $K_{0}(A)$ is defined as direct sum; the equivalence relation $E \oplus \Pi E \backsim 0$ permits us to identify element $-E$ with $\Pi E$.) One can relate $K$-theory also to the theory of differential $\mathbf{Z}_{2}$-graded projective modules, i.e. $\mathbf{Z}_{2}$-graded modules equipped with an odd ( parity reversing) $A$-linear map $Q$, obeying $Q^{2}=0$.We can say that a $\mathbf{Z}_{2}$-graded module $\mathcal{E}$ determines zero element of the group $K_{0}(A)$ iff one can introduce a parity reversing differential $Q$ on $\mathcal{E}$ in such a way that

$$
\operatorname{Im} Q=\operatorname{Ker} Q
$$

(i.e. $Q$ has trivial homology) and $\operatorname{Im} Q$ is a direct summand in $\mathcal{E}$. (In this case we can write that $\mathcal{E}=E \oplus \operatorname{Im} Q$; the map $Q$ identifies $\operatorname{Im} Q$ and $\Pi E$.)

Acknowledgements.

I am deeply indebted to M. Rieffel for numerous consultations on multidimensional noncommutative tori that were thoroughly analyzed in his papers. I am grateful to A. Astashkevich and N. Nekrasov for interesting discussions. I was supported in part by NSF grant DMS 95-00704 and ( during my visit to the Institute for Theoretical Physics, Santa Barbara) by NSF grant PHY94-07194.

REFERENCES.

1. Connes, A., Douglas, M., and Schwarz, A., Noncommutative Geometry and Matrix Theory: Compactification on Tori, hep-th/9711162. Published in JHEP electronic journal.

2. Douglas, M. and Hull, C., D-branes and non-commutative geometry, hep-th/9711165.

Kawano, T. and Okuyama, K., Matrix Theory on Noncommutative Torus, hep-th/9803044.

Cheu, Y.-K. E. and Krogh, M., Noncommutative Geometry from 0branes in a Background B-field, hep-th/9803031.

Berkooz, M., Non-local Field Theories and the Non-commutative Torus, hep-th/980206.

Li, M., Comments on Supersymmetric Yang-Mills Theory on a Noncommutative Torus, hep-th/9802052.

Casalbuoni, R., Algebraic treatment of compactification on noncommutative tori, hep-th/9801170.

Ho, P.-M. and Wu, Y.-S., Noncommutative Gauge Theories in Matrix Theory, hep-th/9801147.

Krogh, M., A Matrix Model for Heterotic $\operatorname{Spin}(32) / Z_{2}$ and Type I String Theory, hep-th/9801034.

Ho, P.-M., Wu, Y.-Y., and Wu, Y.-S. , Towards a Noncommutative Geometric Approach to Matrix Compactification, hep-th/9712201. 
Leigh, R. G. and Rozali, M., A Note on Six-Dimensional Gauge Theories, hep-th/9712168

Aharony, O., Berkooz, M., and Seiberg, N., Light-Cone Description of $(2,0)$ Superconformal Theories in Six Dimensions, hep-th/9712117.

Obers, N. A., Pioline, B., and Rabinovici, E., M-Theory and U-duality on $T^{d}$ with Gauge Backgrounds, hep-th/9712084.

Hull, C. M., U-Duality and BPS Spectrum of Super Yang-Mills Theory and M-Theory, hep-th/9712075.

Blau, M. and O'Laughlin, M., Aspects of U-Duality in Matrix Theory, hep-th/9712047.

3. Rieffel, M. and Schwarz, A., Morita equivalence of multidimensional noncommutative tori, q-alg/9803057.

4.Nekrasov, N. and Schwarz, A., Instantons on noncommutative $R^{4}$, and $(\mathbf{2 , 0 )}$ superconformal six dimensional theory, hep-th/9802068.

5. Astashkevich, A., Nekrasov, N., and Schwarz, A., in preparation.

6. Connes, A., Noncommutative geometry, Academic Press, 1994.

7. Connes, A. and Rieffel, M. Yang-Mills for noncommutative twotori, in : Operator algebras and Mathematical Physics ( Iova City, Iova,1985), pp. 237-266, Contemp. Math. Oper. Algebra. Math.Phys. 62, AMS 1987.

Rieffel, M. A., Induced representations of $C^{*}$-algebras, Advances Math. 13 (1974), 176-257.

Rieffel, M. A., $C^{*}$-algebras associated with irrational rotations, Pacific J. Math. 93 (1981), 415-429.

Rieffel, M. A., Morita equivalence for operator algebras, in "Operator Algebras and Applications" (R. V. Kadison, ed.) Proc. Symp. Pure Math. 38, Amer. Math. Soc., Providence, 1982.

Rieffel, M. A., Projective modules over higher-dimensional non-commutative tori, Canadian J. Math. 40 (1988), 257-338.

Rieffel, M. A., Non-commutative tori - a case study of non-commutative differentiable manifolds, Contemporary Math. 105 (1990), 191-211.

Elliott, G. A., On the K-theory of the $C^{*}$-algebras generated by a projective representation of a torsion-free discrete abelian group, in "Operator Algebras and Group Representations" 157-184, Pitman, London, 1984.

8. Banks, T., Fishler, W., Shenker, S., and Susskind, L., Phys. Rev. D55 (1997) 5112-5128, hep-th /9610043

9. Ishibashi, N.Kawai, H., Kitazava, I., and Tsuchiya, A., Nucl. Phys. B492 (1997) 467-491

10.Konechny, A and Schwarz, A., in preparation. 